\title{
Decrease in hospital admissions for respiratory diseases during the COVID-19 pandemic: a nationwide claims study
}

\author{
Kyungmin Huh, ${ }^{1}$ Young-Eun Kim, ${ }^{2}$ Wonjun Ji, ${ }^{3}$ Dong Wook Kim, ${ }^{2}$ Eun-Joo Lee, ${ }^{2}$ \\ Jong-Hun Kim, ${ }^{4}$ Ji-Man Kang, ${ }^{5}$ Jaehun Jung ${ }^{6,7}$
}

For numbered affiliations see end of article.

\section{Correspondence to}

Dr Jaehun Jung, Artificial Intelligence and Big Data Convergence Center, Gachon University Gil Medical Center, Incheon, Republic of Korea; eastside1st@gmail.com

$\mathrm{KH}, \mathrm{Y}$-EK and WJ contributed equally.

Received 4 November 2020 Revised 22 January 2021 Accepted 1 February 2021

\section{Linked}

- http://dx.doi.org/10.1136/ thoraxjnl-2020-216512

- http://dx.doi.org/10.1136/ thoraxjnl-2020-216380

\section{Check for updates}

(C) Author(s) (or their employer(s)) 2021. No commercial re-use. See rights and permissions. Published by BMJ.

To cite: Huh K, Kim Y-E, Ji W, et al. Thorax Epub ahead of print: [please include Day Month Year]. doi:10.1136/ thoraxjnl-2020-216526

\section{ABSTRACT}

Non-pharmaceutical interventions (NPIs) have been widely implemented to mitigate the spread of COVID-19. We assessed the effect of NPIs on hospitalisations for pneumonia, influenza, COPD and asthma. This retrospective, ecological study compared the weekly incidence of hospitalisation for four respiratory conditions before (January 2016-January 2020) and during (February-July 2020) the implementation of NPI against COVID-19. Hospitalisations for all four respiratory conditions decreased substantially during the intervention period. The cumulative incidence of admissions for COPD and asthma was $58 \%$ and $48 \%$ of the mean incidence during the 4 preceding years, respectively.

\section{INTRODUCTION}

Non-pharmaceutical interventions (NPIs) have been the most important public health measure to mitigate the impact of the COVID-19 pandemic. NPIs implemented against COVID-19 are expected to reduce the transmission of other respiratory viruses, and early studies have reported a decrease in the incidence of viral respiratory infections. ${ }^{12}$

Thus, we assessed the incidence of hospitalisations due to pneumonia, influenza and chronic lung diseases during the COVID-19 pandemic in South Korea and compared it with the previous seasonal average and temporal trends using nationwide healthcare claims database.

\section{MATERIALS AND METHODS \\ Data source}

The daily number of hospitalisations was obtained from the National Health Insurance Service (NHIS) claims database. South Korea has a universal, singlepayer healthcare coverage for all residents, and all covered costs are reimbursed by the NHIS. The cause of admission was captured from the primary diagnosis code using the International Statistical Classification of Diseases and Related Health Problems, 10th edition. The weekly incidence of hospitalisations was calculated by dividing the weekly number of admissions by the annual mid-year population, obtained from the Korean Statistical Information Service (http://kosis.kr).

\section{Study design and statistical analysis}

This was a retrospective ecological study comparing the incidence of hospitalisation due to acute respiratory infections (pneumonia and influenza) and chronic respiratory diseases (COPD and asthma) before and during the COVID-19 pandemic. We also compared hospitalisations with the primary diagnosis of diabetic ketoacidosis or hyperosmolar hyperglycaemic state (DKA/HHS), intracranial haemorrhage (ICH), myocardial infarct (MI) and cancer to account for change in healthcare utilisation.

The baseline period was defined as January 2016 to January 2020 and the intervention period as February to July 2020. Prediction models were constructed for each disease based on the incidence during the entire baseline period using an autoregressive integrated moving average (ARIMA). Parameters for the ARIMA models were selected using the 'auto.arima' function in the 'forecast' package for R software (https://pkg.robjhyndman. com/forecast/). The weekly incidence of hospitalisations during the intervention period was visually compared with the $95 \%$ CI of the predicted values.

Furthermore, the cumulative incidence (CuI) during the intervention period (ie, sum of weekly incidences during the period) was compared with the 4-year mean $\mathrm{CuI}$ during the same months (February-July) of the baseline period. The difference in CuI was tested using a Poisson test. All tests were two-tailed and $\mathrm{p}$ values $<0.05$ were considered statistically significant. Statistical analyses were performed using R V.3.6.2 (R Foundation for Statistical Computing, Vienna, Austria).

\section{RESULTS}

Hospitalisations with the primary diagnosis of pneumonia decreased substantially during the intervention period (figure 1). The $\mathrm{CuI}$ during the intervention period was 0.47 times the $\mathrm{CuI}$ of the 4 -year mean $(95 \%$ CI 0.45 to $0.50 ; \mathrm{p}<0.001$; table 1 ). The weekly admissions for influenza were within 95\% CI of the predicted values; however, the $\mathrm{CuI}$ was significantly lower than the 4-year mean (0.22 times the 4 -year mean; $95 \%$ CI 0.19 to 0.25 ; $\mathrm{p}<0.001)$. Hospitalisations due to pneumonia or influenza have decreased to comparable proportions among patients with pre-existing COPD or asthma.

Hospital admissions due to COPD and asthma also decreased substantially. The CuIs for COPD and asthma admissions were 0.58 times $(95 \% \mathrm{CI}$ 0.49 to $0.68 ; \mathrm{p}<0.001)$ and 0.48 times $(95 \% \mathrm{CI}$ 0.39 to $0.57 ; \mathrm{p}<0.001)$ the mean baseline CuIs, respectively. The trend was consistent across all sex 

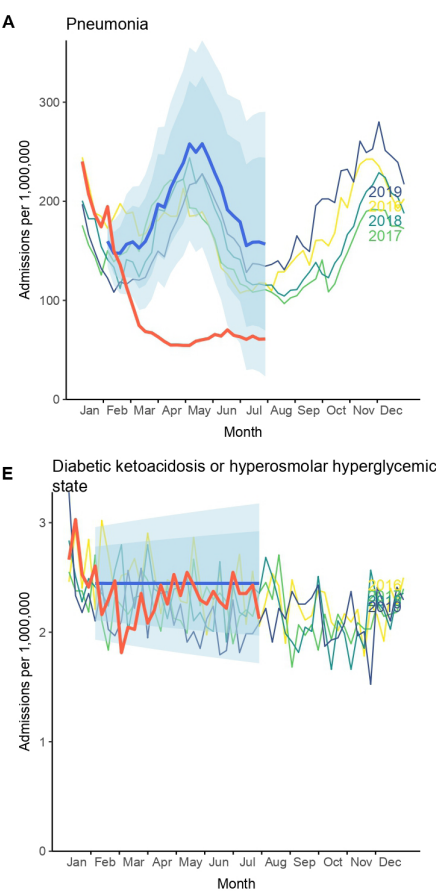
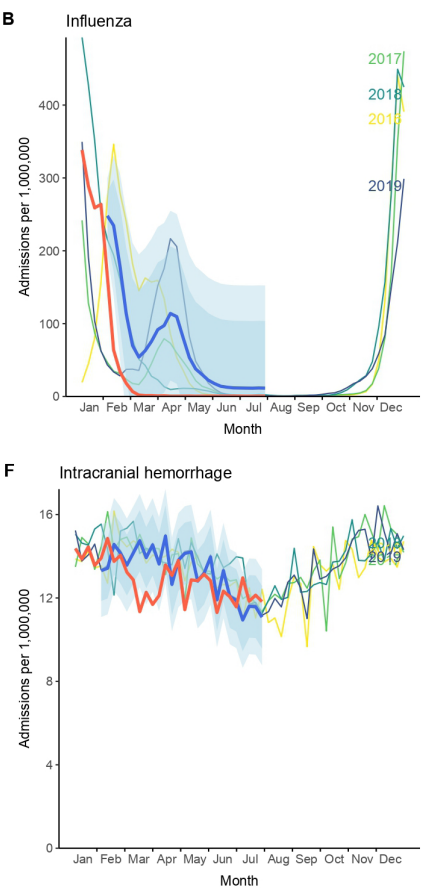
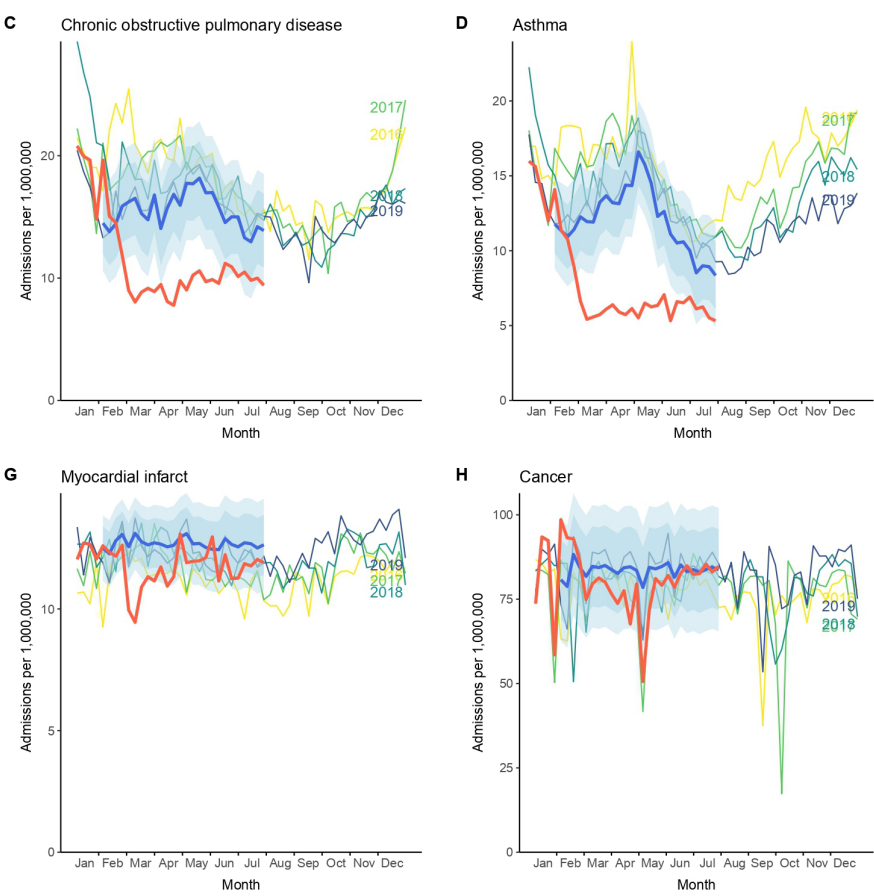

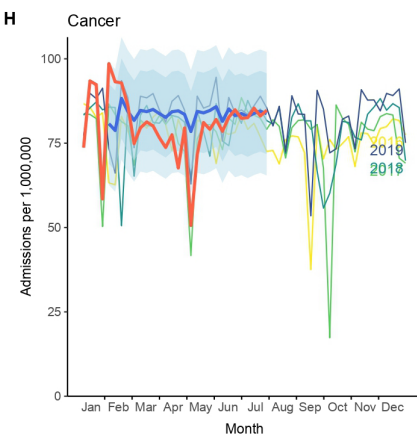

Figure 1 Weekly incidence of hospital admission per 1000000 population by primary diagnosis. Bold red lines denote the actual incidence during the intervention period; bold blue lines denote the predicted incidence; and dark and light blue shades denote the $80 \%$ and $95 \%$ Cls of the predicted incidence, respectively. Panels A-D show the hospitalisations due to respiratory diseases; panels $\mathrm{E}-\mathrm{H}$, hospitalisations for non-respiratory acute (E-G) and chronic $(\mathrm{H})$ conditions.

and age groups (data not shown).

In contrast, hospital admissions for other acute (DKA/HHS, ICH and $\mathrm{MI}$ ) and chronic (cancer) conditions during the intervention period were not significantly different from the predicted values or the 4-year means. Transient short-term decreases in admissions were observed from March through May 2020, but these 'dips' were not sustained and there was no significant difference in the CuI between the intervention and the baseline period (table 1).

\section{DISCUSSION}

Influenza and a substantial portion of pneumonia are primarily mediated through droplet transmission. Personal hygiene and droplet precautions are well-known effective prevention measures for these infections. ${ }^{3}$ Since the early days of the COVID-19 epidemic, South Korea has thoroughly carried out social distancing, personal hygiene and universal use of face masks. ${ }^{4}$ In this study, the significant decrease in hospital admissions for influenza, pneumonia, COPD and asthma suggests the unintended benefits of these measures. This finding is consistent with previous reports that showed a decreased incidence of respiratory infections and influenza. ${ }^{125}$

It is noteworthy that not only the admissions for respiratory infections but also the overall admissions for COPD and asthma decreased substantially. Similar observations have been reported from Europe and the USA during the early stage of the COVID-19 epidemic. ${ }^{6-8}$ Our results reaffirm those findings; also, our findings are unique as South Korea did not undergo 'lockdown' as many

Table 1 Cumulative incidence of hospital admission from February through July in 2020 versus 2016-2019

\begin{tabular}{|c|c|c|c|c|}
\hline \multirow[b]{2}{*}{ Disease } & \multicolumn{2}{|c|}{$\begin{array}{l}\text { Cumulative incidence, February-July } \\
\text { (per } 1000000 \text { ) }\end{array}$} & \multirow[b]{2}{*}{ Rate ratio $(95 \% \mathrm{Cl})$} & \multirow[b]{2}{*}{$P$ value } \\
\hline & $\begin{array}{l}\text { Mean } \\
(2016-2019)\end{array}$ & Observed (2020) & & \\
\hline Pneumonia & 3965.29 & 1872.59 & $0.47(0.45$ to 0.50$)$ & $<0.001$ \\
\hline Patients with COPD & 28304.95 & 15182.24 & $0.54(0.53$ to 0.55$)$ & $<0.001$ \\
\hline Patients with asthma & 3196.58 & 1200.35 & $0.38(0.35$ to 0.40$)$ & $<0.001$ \\
\hline Influenza & 1366.09 & 299.33 & 0.22 (0.19 to 0.25$)$ & $<0.001$ \\
\hline Patients with COPD & 1355.74 & 363.06 & $0.27(0.24$ to 0.30$)$ & $<0.001$ \\
\hline Patients with asthma & 554.65 & 79.56 & $0.14(0.11$ to 0.18$)$ & $<0.001$ \\
\hline COPD & 435.11 & 251.70 & $0.58(0.49$ to 0.68$)$ & $<0.001$ \\
\hline Asthma & 353.16 & 168.13 & 0.48 (0.39 to 0.57$)$ & $<0.001$ \\
\hline Diabetic ketoacidosis/hyperosmolar hyperglycaemic state & 54.94 & 55.25 & 1.02 (0.69 to 1.51$)$ & $>0.999$ \\
\hline Intracranial haemorrhage & 329.17 & 305.69 & 0.93 (0.79 to 1.09$)$ & 0.361 \\
\hline Myocardial infarct & 286.37 & 280.00 & $0.98(0.82$ to 1.15$)$ & 0.801 \\
\hline Cancer & 1914.28 & 1922.59 & $1.00(0.94$ to 1.07$)$ & 0.910 \\
\hline
\end{tabular}


European countries did and we demonstrated that the decrease was sustained for at least 6 months. Conversely, hospital admissions due to other acute (DKA/HHS, ICH and MI) and chronic (cancer) conditions did not decrease, which shows this was not attributable to an alteration in healthcare-seeking behaviour. Our findings suggest that the decrease in admissions due to COPD and asthma might be associated with the decrease in respiratory infections, which are the most common triggers for acute exacerbation of COPD and asthma. ${ }^{9} 10$

This study has some limitations. First, we identified the cause of admission from the primary diagnosis code used for billing. However, there is no reason to suspect a change of coding practices during the intervention period. Second, we could not directly confirm from the claims database that the reduction in respiratory infections led to the decrease in admissions among patients with COPD and asthma. There remains a possibility that the decrease in admissions resulted from the alteration in healthcare-seeking behaviour, although no significant change was observed in admissions for non-respiratory conditions. In such case, the decrease in hospitalisation may lead to delayed care and increased mortality later.

In conclusion, we found that the hospital admission rates for respiratory infections and chronic lung diseases decreased significantly after the implementation of NPIs against COVID-19, despite the lack of change in admission rates for non-respiratory conditions.

\section{Author affiliations}

'Division of Infectious Diseases, Department of Medicine, Samsung Medical Center, Sungkyunkwan University School of Medicine, Seoul, Republic of Korea

${ }^{2}$ Department of Big Data Strategy, National Health Insurance Service, Wonju, Republic of Korea

${ }^{3}$ Department of Pulmonary and Critical Care Medicine, Asan Medical Center, University of Ulsan College of Medicine, Seoul, Republic of Korea

${ }^{4}$ Department of Social and Preventive Medicine, Sungkyunkwan University School of Medicine, Suwon, Republic of Korea

${ }^{5}$ Department of Pediatrics, Severance Children's Hospital, Yonsei University College of Medicine, Seoul, Republic of Korea

${ }^{6}$ Artificial Intelligence and Big Data Convergence Center, Gachon University Gil Medical Center, Incheon, Republic of Korea

${ }^{7}$ Department of Preventive Medicine, Gachon University College of Medicine, Incheon, Republic of Korea

Acknowledgements This study used the National Health Information Database (NHIS-2020-1-572) made by the National Health Insurance Service (NHIS).

Contributors $\mathrm{KH}, \mathrm{WJ}$ and JJ had full access to the study data and take responsibility for the integrity and accuracy of the analysis. Concept and design: $\mathrm{KH}$, JJ. Acquisition, analysis or interpretation of data: KH, WJ, Y-EK, E-JL, DWK. Drafting of the manuscript: KH, JJ, WJ. Critical review of the manuscript: J-HK, J-MK. Statistical analysis: KH, JJ, Y-EK.
Funding This study was supported by grants from the Gachon University Gil Medical Center (grant numbers 2018-17 and 2019-11). The sponsor of the study was not involved in study design, analysis and interpretation of data; writing of the report; or the decision to submit the study results for publication.

Competing interests None declared.

Patient consent for publication Not required.

Ethics approval The research was conducted ethically in accordance with the World Medical Association Declaration of Helsinki and was approved by the appropriate institutional review board of the Gachon University College of Medicine, Incheon, Republic of Korea (GFIRB2020-384).

Provenance and peer review Not commissioned; externally peer reviewed.

This article is made freely available for use in accordance with BMJ's website terms and conditions for the duration of the covid-19 pandemic or until otherwise determined by BMJ. You may use, download and print the article for any lawful, non-commercial purpose (including text and data mining) provided that all copyright notices and trade marks are retained.

\section{ORCID iD}

Jaehun Jung http://orcid.org/0000-0002-4856-3668

\section{REFERENCES}

1 Tan JY, Conceicao EP, Sim XYJ, et al. Public health measures during COVID-19 pandemic reduced hospital admissions for community respiratory viral infections. J Hosp Infect 2020;106:387-9.

2 Huh K, Jung J, Hong J, et al. Impact of non-pharmaceutical interventions on the incidence of respiratory infections during the COVID-19 outbreak in Korea: a nationwide surveillance study. Clin Infect Dis 2020. doi:10.1093/cid/ciaa1682. [Epub ahead of print: 05 Nov 2020].

3 Suess T, Remschmidt C, Schink SB, et al. The role of facemasks and hand hygiene in the prevention of influenza transmission in households: results from a cluster randomised trial; Berlin, Germany, 2009-2011. BMC Infect Dis 2012;12:26.

4 COVID-19 National Emergency Response Center, Epidemiology \& Case Management Team, Korea Centers for Disease Control \& Prevention. Contact transmission of COVID-19 in South Korea: novel investigation techniques for tracing contacts. Osong Public Health Res Perspect 2020;11:60-3.

5 Nolen LD, Seeman S, Bruden D, et al. Impact of social distancing and travel restrictions on non-COVID-19 respiratory hospital admissions in young children in rural Alaska. Clin Infect Dis 2020. doi:10.1093/cid/ciaa1328. [Epub ahead of print: 05 Sep 2020].

6 Krivec U, Kofol Seliger A, Tursic J. COVID-19 lockdown dropped the rate of paediatric asthma admissions. Arch Dis Child 2020;105:809-10.

7 Berghaus TM, Karschnia P, Haberl S, et al. Disproportionate decline in admissions for exacerbated COPD during the COVID-19 pandemic. Respir Med 2020:106120.

8 Birkmeyer JD, Barnato A, Birkmeyer N, et al. The impact of the COVID-19 pandemic on hospital admissions in the United States. Health Aff 2020;39:2010-7.

9 Papi A, Bellettato CM, Braccioni F, et al. Infections and airway inflammation in chronic obstructive pulmonary disease severe exacerbations. Am J Respir Crit Care Med 2006;173:1114-21

10 Masoli M, Fabian D, Holt S, et al. The global burden of asthma: Executive summary of the GINA dissemination Committee report. Allergy 2004;59:469-78. 